\title{
A peptide antigen derived from EGFR T790M is immunogenic in non-small cell lung cancer
}

\author{
KAZUYA OFUJI $^{1,2}$, YOSHITAKA TADA ${ }^{1,3}$, TOSHIAKI YOSHIKAWA ${ }^{1}$, MANAMI SHIMOMURA ${ }^{1}$, \\ MAYUKO YOSHIMURA ${ }^{1}$, KEIGO SAITO ${ }^{1}$, YASUNARI NAKAMOTO ${ }^{2}$ and TETSUYA NAKATSURA ${ }^{1}$ \\ ${ }^{1}$ Division of Cancer Immunotherapy, Exploratory Oncology Research and Clinical Trial Center, National Cancer \\ Center, Kashiwa, Chiba; ${ }^{2}$ Second Department of Internal Medicine, Faculty of Medical Sciences, University of \\ Fukui, Fukui; ${ }^{3}$ Research Institute for Biomedical Sciences, Tokyo University of Science, Noda, Chiba, Japan
}

Received August 29, 2014; Accepted October 9, 2014

DOI: 10.3892/ijo.2014.2787

\begin{abstract}
Lung cancer is the leading cause of cancer-related deaths worldwide. Epidermal growth factor receptor-tyrosine kinase inhibitors (EGFR-TKIs), such as gefitinib and erlotinib, have demonstrated marked clinical activity against non-small cell lung cancer (NSCLC) harboring activating epidermal growth factor receptor (EGFR) mutations. However, in most cases, patients develop acquired resistance to EGFR-TKI therapy. The threonine to methionine change at codon 790 of EGFR (EGFR T790M) mutation is the most common acquired resistance mutation, and is present in $\sim 50 \%$ cases of TKI resistance. New treatment strategies for NSCLC patients harboring the EGFR T790M mutation are required. We evaluated the immunogenicity of an antigen derived from EGFR with the T790M mutation. Using BIMAS we selected several EGFR T790M-derived peptides bound to human leukocyte antigen (HLA)-A*02:01. T790M-A peptide (789-797) (IMQLMPFGC)-specific cytotoxic T lymphocytes (CTLs) were induced from peripheral blood mononuclear cells (PBMCs) of HLA-A2 ${ }^{+}$healthy donors. An established T790M-A-specific CTL line showed reactivity against the NCSLC cell line, H1975-A2 (HLA-A2 ${ }^{+}$, T790M $^{+}$), but not H1975 (HLA-A2-, T790M+), and the corresponding
\end{abstract}

Correspondence to: Professor Tetsuya Nakatsura, Division of Cancer Immunotherapy, Exploratory Oncology Research and Clinical Trial Center, National Cancer Center, 6-5-1 Kashiwanoha, Kashiwa, Chiba 277-8577, Japan

E-mail: tnakatsu@east.ncc.go.jp

Abbreviations: aAPC, artificial antigen-presenting cell; ELISPOT, enzyme-linked immuno spot; HLA, human leukocyte antigen; IFN- $\gamma$, interferon- $\gamma$; MAPK, mitogen-activated protein kinase; PBMC, peripheral blood mononuclear cell; PI3K, phosphatidylinositol 3-kinase; PFS, progression-free survival; STAT, signal transducer and activator of transcription

Key words: acquired resistance, CTL epitope, EGFR T790M, immunotherapy, non-small cell lung cancer wild-type peptide (ITQLMPFGC)-pulsed T2 cells using an interferon- $\gamma($ IFN- $\gamma)$ enzyme-linked immuno spot (ELISPOT) assay. This CTL line also demonstrated peptide-specific cytotoxicity against H1975-A2 cells. This finding suggests that the EGFR T790M mutation-derived antigen could be a new target for cancer immunotherapy.

\section{Introduction}

Lung cancer is the leading cause of cancer-related deaths worldwide (1). Non-small cell lung cancer (NSCLC) accounts for $\sim 80 \%$ of all lung cancer cases. Despite recent development in treatment agents, the prognosis for lung cancer patients remains poor (2).

Overexpression of epidermal growth factor receptor (EGFR) is observed in various malignancies, including lung cancer (3). EGFR activation induces many intracellular signaling pathways, such as the mitogen-activated protein kinase (MAPK), phosphatidylinositol 3-kinase (PI3K), and signal transducer and activator of transcription (STAT) pathways, which cause tumor cell proliferation and survival (4). The EGFR pathway is an appropriate target for cancer therapy, and several agents that block this pathway have been developed. In particular, epidermal growth factor receptor-tyrosine kinase inhibitors (EGFR-TKIs), such as gefitinib and erlotinib, demonstrated marked clinical activity against NSCLC harboring an activating EGFR mutation (5-9). However, patients develop acquired resistance to EGFR-TKIs almost without exception (10). A secondary mutation, resulting in a threonine to methionine change at codon 790 of EGFR (EGFR T790M), is the major mechanism of EGFR-TKI resistance $(10,11)$. Additionally, some reports suggest that the EGFR T790M mutation may not be rare and may exist in a small population of in tumor cells before TKI treatment (12-14). Moreover, a pre-existing T790M mutation was associated with shorter progression-free survival (PFS) in patients receiving TKI treatment $(13,14)$. At this time, no standard treatment for EGFR mutant patients with acquired resistance has yet been established, and novel strategies for overcoming this resistance issue are required.

Immunotherapy for NSCLC patients is considered to be a potentially feasible option, because of its high specificity 
and low toxicity against normal tissues; indeed, several tumor-associated antigen (TAA)-targeted phase $2 / 3$ studies are ongoing (15). However, unfortunately, the results of a TAA-based vaccine therapy study were unsatisfactory (16). One concept for improving the effect of cancer vaccine therapy is to target mutated antigen-derived epitopes. It has been reported that various mutated epitopes were recognized by tumor-reactive T cells $(17,18)$, suggesting that the mutated epitope was potentially immunogenic and thus might function as an immunotherapeutic target. There are few studies of immunotherapy targeting the EGFR T790M mutation. Here, we hypothesized that EGFR T790M-harboring cancer cells could be targeted by activated immune cells, and attempted to assess the immunogenicity of the EGFR T790M mutation-derived antigen in vitro. In the present study, we identified the human leukocyte antigen (HLA)-A2-restricted EGFR T790M mutation-derived epitope. Our results suggest that immunotherapy targeting the EGFR T790M mutation-derived antigen may be a novel treatment option for NSCLC patients with the T790M mutation. The combination of immunotherapy and EGFR-TKI therapy also may be a novel strategy for prevention of T790M-mediated resistance.

\section{Materials and methods}

Cell lines. The human NSCLC cell line H1975 was provided by Professor Seiji Yano (Kanazawa University, Ishikawa, Japan). H1975-A2 (H1975 transfected with HLA-A2) was provided by Dr Tetsuro Sasada (Kurume University, Fukuoka, Japan). Artificial APC-A2 (aAPC-A2) cells, which were generated by transduction of HLA-A*02:01, CD80, and CD83 molecules into K562 cells, were provided by Dr Naoto Hirano (Dana-Farber Cancer Institute, Boston, MA, USA). T2 cells (HLA-A*02:01, TAP ${ }^{*}$ ) and human NSCLC cell line 11-18 were purchased from Riken (Saitama, Japan). These cell lines were cultured in RPMI-1640 (Sigma Chemical Co., St. Louis, MO, USA), supplemented with $10 \%$ FBS (Gibco-BRL, Carlsbad, CA, USA), $100 \mathrm{U} / \mathrm{ml}$ penicillin, and $100 \mu \mathrm{g} / \mathrm{ml}$ streptomycin in a humidified atmosphere containing $5 \% \mathrm{CO}_{2}$.

PBMC collection. Peripheral blood samples were collected from four HLA-A*02:01-positive healthy donors, after informed consent was obtained. Peripheral blood mononuclear cells (PBMCs) were isolated by density centrifugation using Ficoll-Hypaque (Pharmacia, Uppsala, Sweden) and frozen in liquid nitrogen until use.

Epitope prediction and synthesis. The epitope prediction software BIMAS (http://www-bimas.cit.nih.gov/molbio/hla_bind/) was used to predict peptides that could bind to HLA-A2. EGFR T790M mutation-derived peptides (purity $>95 \%$ ) were purchased from Scrum, Inc. (Tokyo, Japan). H-2 Kb-restricted ovalbumin (OVA) (257-264) (SIINFEKL) peptide (AnaSpec, Inc., Fremont, CA, USA) was used as a negative control in the peptide-binding assay. HLA-A2-restricted cytomegalovirus (CMV) (495-503) (NLVPMVATV) peptide was used as a positive control peptide, and an HLA-A2-restricted HIV-gag (77-85) (SLYNTYATL) peptide (American Peptide Company, Sunnyvale, CA, USA) as an irrelevant peptide in cytotoxic T lymphocyte (CTL) assays.
Peptide-binding assay. After incubation in culture medium at $26^{\circ} \mathrm{C}$ overnight, T2 cells were washed with PBS and suspended in $1 \mathrm{ml}$ Opti-MEM (Invitrogen Life Technologies, Carlsbad, CA, USA) with peptide $(100 \mu \mathrm{g} / \mathrm{ml})$, followed by incubation at $26^{\circ} \mathrm{C}$ for $3 \mathrm{~h}$ and then at $37^{\circ} \mathrm{C}$ for $2.5 \mathrm{~h}$. After washing with PBS, HLA-A2 expression was measured using a BD FACSCanto II flow cytometer (BD Biosciences, San Jose, CA, USA) using a FITC-conjugated HLA-A2 (MBL Co., Ltd., Aichi, Japan)-specific monoclonal antibody. Mean fluorescence intensity (MFI) was analyzed using the FlowJo software (Tomy Digital Biology Co., Ltd., Tokyo, Japan). An OVA peptide was used as a negative control. A CMV peptide was used as a positive control peptide.

Generation of DCs. CD14+ cells were isolated from PBMCs using human CD14 microbeads (Miltenyi Biotec $\mathrm{GmbH}$, Bergisch Gladbach, Germany). Immature dendritic cells (DCs) were generated from CD14 ${ }^{+}$cells using IL-4 (10 ng/ml; PeproTech, Inc., Rocky Hill, NJ, USA) and granulocyte-macrophage colony-stimulating factor (GM-CSF) (10 ng/ml; PeproTech, Inc.) in RPMI-1640 supplemented with $10 \%$ FBS. Maturation of DCs was induced by prostaglandin E2 (PGE2) $(1 \mu \mathrm{g} / \mathrm{ml}$; Sigma Chemical Co.) and tumor necrosis factor- $\alpha$ (TNF- $\alpha$ ) (10 ng/ml; PeproTech, Inc.).

Induction of peptide-specific CTLs. CD8 ${ }^{+}$cells were isolated using human CD8 microbeads (Miltenyi Biotec $\mathrm{GmbH})$ from PBMCs. $\mathrm{CD}^{+}$cells $\left(2 \times 10^{6}\right.$ cells/well $)$ were stimulated with peptide-pulsed $(10 \mu \mathrm{g} / \mathrm{ml})$ 100-Gy-irradiated autologous mature DCs $\left(1 \times 10^{5}\right.$ cells/well) in RPMI-1640 containing $10 \%$ heat-inactivated human AB serum. After 1 week, these cells were stimulated twice weekly with peptide-pulsed $(10 \mu \mathrm{g} / \mathrm{ml})$ 200-Gy-irradiated aAPC-A2 cells $\left(1 \times 10^{5}\right.$ cells/well $)$. Supplementation with $10 \mathrm{IU} / \mathrm{ml}$ IL-2 (Proleukin; Novartis, Basel, Switzerland) and $10 \mathrm{ng} / \mathrm{ml}$ IL-15 (PeproTech, Inc.) was performed at 3-4-day intervals between stimulations.

IFN- $\gamma$ ELISPOT assay. Specific secretion of interferon- $\gamma($ IFN- $\gamma)$ from human CTLs in response to stimulator cells was assayed using the IFN- $\gamma$ enzyme-linked immuno spot (ELISPOT) kit (BD Biosciences), according to the manufacturer's instructions. Stimulator cells were pulsed with peptide for $2 \mathrm{~h}$ at room temperature and then washed three times. Responder cells were incubated with stimulator cells for $20 \mathrm{~h}$. The resulting spots were counted using an ELIPHOTO counter (Minerva Tech, Tokyo, Japan).

CD107a assay and generation of a CTL line. CD8 ${ }^{+}$cells isolated using human CD8 microbeads from cultured cells were incubated with peptide-pulsed $\mathrm{T} 2$ cells at a ratio of 2:1 for $3.5 \mathrm{~h}$ at $37^{\circ} \mathrm{C}$. CD107a-specific antibodies (BD Biosciences) were included in the mixture during the incubation period. $\mathrm{CD}^{+} \mathrm{CD} 107 \mathrm{a}^{+}$cells were sorted using a FACSAria II cell sorter (BD Biosciences). Sorted CTLs were stimulated, and the CTL line was established as described previously (19).

Cytotoxicity assay. Cytotoxic capacity was analyzed using the Terascan VPC system (Minerva Tech). The CTL line 
Table I. Predicted EGFR T790M-derived peptides binding to HLA-A2.

\begin{tabular}{lcccc}
\hline Peptide name & Position & Length & Sequence & BIMAS score $^{\mathrm{a}}$ \\
\hline T790M-A & $789-797$ & 9 & IMQLMPFGC & 35.378 \\
T790M-B & $790-799$ & 10 & MQLMPFGCLL & 51.77 \\
T790M-C & $788-797$ & 10 & LIMQLMPFGC & 24.921 \\
T790M-D & $789-797^{\mathrm{b}}$ & 9 & IMQLMPFGV & 495.288 \\
T790M-E & $789-797^{\mathrm{c}}$ & 9 & IMQLMPFGL & 152.124 \\
T790M-Awt & $789-797$ & 9 & ITQLMPFGC & 0.68 \\
\hline
\end{tabular}

äBinding scores were estimated using the BIMAS software (http://www-bimas.cit.nih.gov/molbio/hla_bind/). ${ }^{\text {b,c }}$ The cysteine (C) residue at position 797 was mutated to valine (V) and leucine (L), respectively. EGFR T790M, threonine to methionine change at codon 790 of EGFR.

was used as the effector cell type. Target cells were labeled in calcein-AM (Dojindo Molecular Technologies, Inc., Kumamoto, Japan) solution for $30 \mathrm{~min}$ at $37^{\circ} \mathrm{C}$. The labeled cells were then co-cultured with the effector cells for 4-6 h. Fluorescence intensity was measured before and after the culture period, and specific cytotoxic activity was calculated using the following formula: $\%$ cytotoxicity $=\{1-[$ (average fluorescence of the sample wells - average fluorescence of the maximal release control wells)/(average fluorescence of the minimal release control wells - average fluorescence of the maximal release control wells)]\} x $100 \%$.

\section{Results}

Assessment of EGFR T790M-derived peptide binding to HLA-A*02:01 molecules. As the candidates of HLA-A*02:01restricted EGFR T790M-derived CTL epitopes, we selected five 9- or 10-mer peptides with high predicted HLA-A*02:01-binding scores, calculated using BIMAS software. Three of the five EGFR T790M-derived peptides had higher binding scores than the corresponding wild-type peptides. Some studies have reported that modified peptides with single amino acid substitutions exhibit improved affinity for HLA molecules and enhanced immunogenicity (20-22); thus, we also designed two modified peptides. These modified peptides with a substitution of Cys for Val (T790M-D) or Leu (T790M-E) at codon 797 showed higher binding scores (Table I).

Using the HLA-A2 TAP-deficient T2 cell line, the binding affinity of the five synthetic peptides to HLA-A2 was assessed. A peptide-binding assay showed that three EGFR T790M-derived peptides were able to bind to HLA-A*02:01 molecules. In particular, the binding capability of the T790M-A peptide to HLA-A*02:01 molecules was higher than that of the corresponding wild-type peptide. This result suggests that the single amino acid substitution at codon 790 improved the binding affinity for HLA-A*02:01 molecules. The binding affinities of two mutated peptides (T790M-D and -E) to HLA-A*02:01 were equivalent to that of the CMV peptide used as a positive control (Fig. 1).

Induction of EGFR T790M-derived peptide-specific CTLs from human PBMCs. To evaluate the immunogenic potential of the five predicted HLA-A*02:01-binding peptides derived from EGFR T790M, we attempted to induce peptide-specific

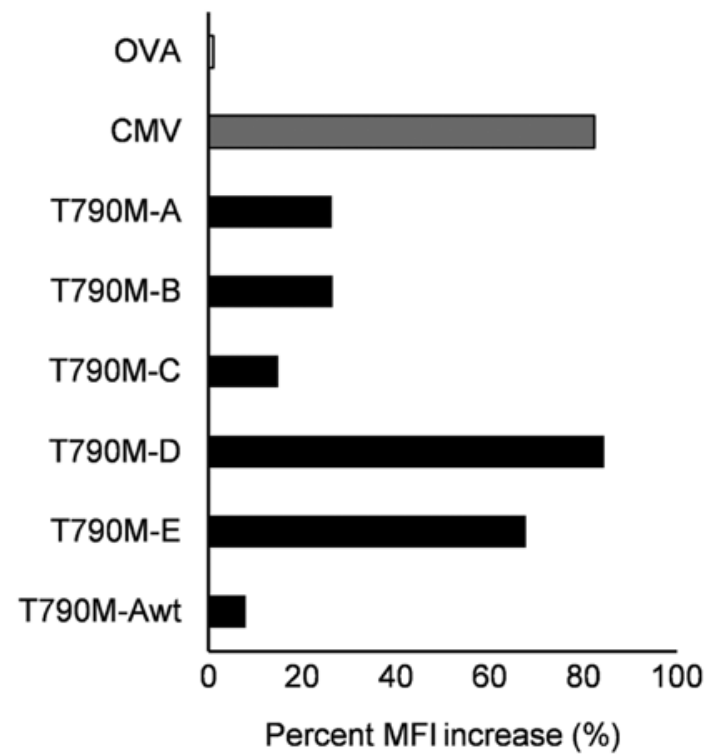

Figure 1. Binding of threonine to methionine change at codon 790 of EGFR (EGFR T790M)-derived peptides to human leukocyte antigen (HLA)-A2 molecule. A T2 binding assay was performed using a FACS system. An ovalbumin (OVA) peptide was used as a negative control. The bars show percent increases in mean fluorescence intensity (MFI). The average of two independent experiments is shown. (Percent MFI increase $)=($ MFI with the given peptide - MFI without peptide $) /($ MFI without peptide) $\mathrm{x} 100$.

CTLs from human PBMCs obtained from four healthy donors. Several reports have shown the usefulness of artificial antigen-presenting cells (aAPCs) for the induction and expansion of peptide-specific CTLs from PBMCs $(23,24)$. Thus, we attempted to induce such CTLs using aAPCs. CD8 ${ }^{+}$cells were isolated from human PBMCs using human CD8 microbeads, and then stimulated with peptide-pulsed DCs for 1 week and subsequently, stimulated twice weekly with peptide-pulsed aAPC-A2 (Fig. 2A). As shown in Fig. 2B, ELISPOT assays revealed that T790M-A (789-797) (IMQLMPFGC)-specific CTLs were induced from PBMCs from all four donors. Also, induction of T790M-B (790-799) (MQLMPFGCLL)-specific CTLs were induced from PBMCs from two of the four healthy donors. However, stimulation with three other peptides, including modified peptides, did not induce peptide-specific CTLs. These results suggest that T790M-A (789-797) and T790M-B (790-799) have immunogenic potential and that 


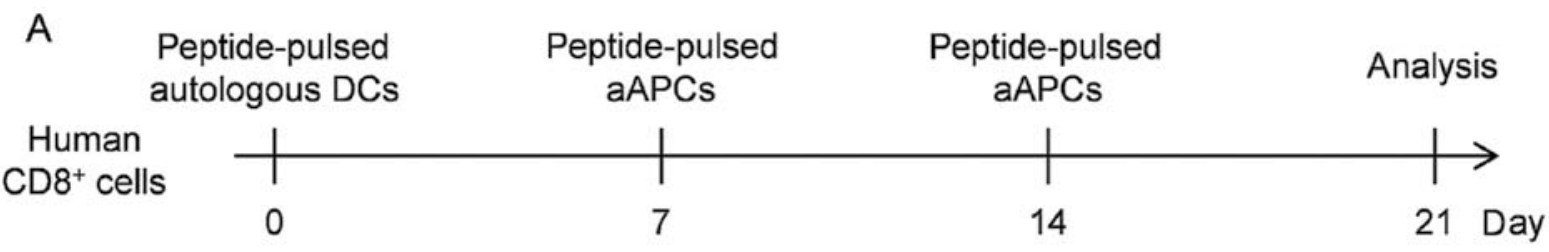

B

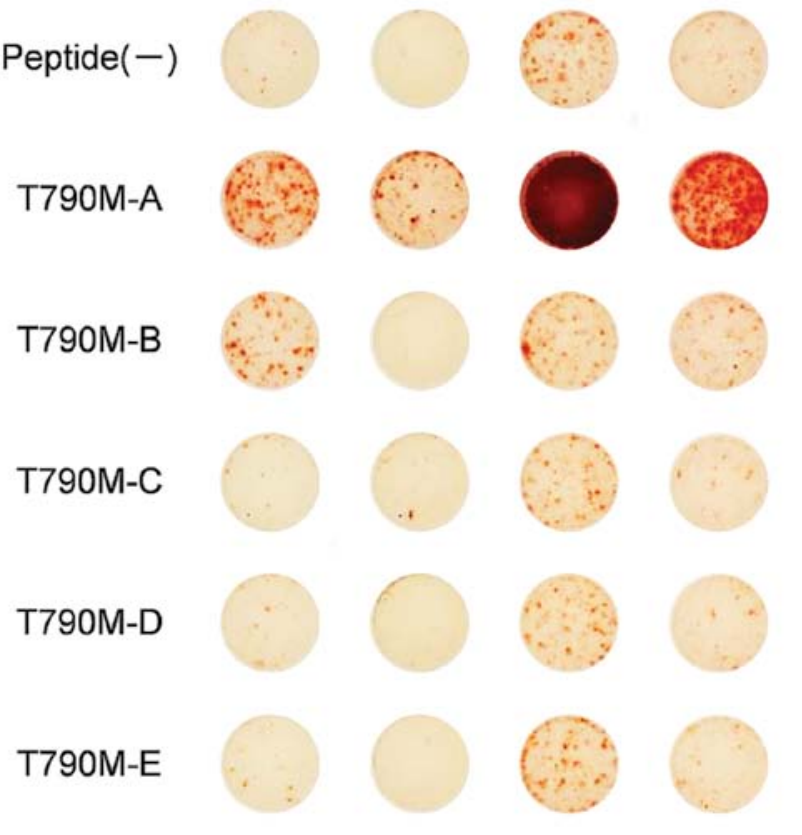

C

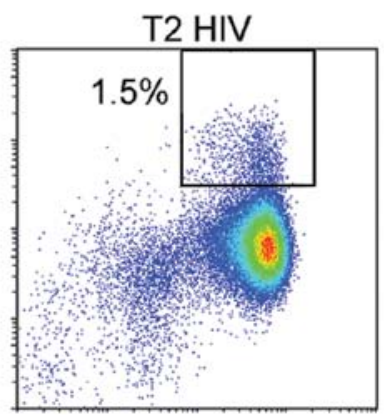

T2 T790M-A

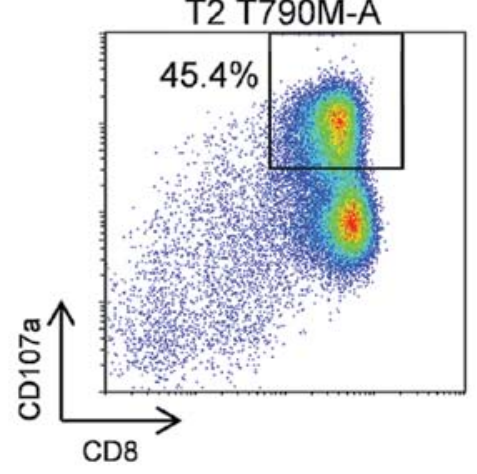

Figure 2. Induction of threonine to methionine change at codon 790 of EGFR (EGFR T790M)-derived peptide-specific cytotoxic T lymphocytes (CTLs) from peripheral blood mononuclear cells (PBMCs) of healthy donors. (A) Induction schedule of peptide-specific CTLs. CD8 ${ }^{+}$cells $\left(2 \times 10^{6}\right.$ cells) isolated by anti-human CD8 microbeads from PBMCs were incubated with $10 \mu \mathrm{g} / \mathrm{ml}$ peptide-pulsed autologous dendritic cells (DCs) $\left(1 \times 10^{5}\right.$ cells) on day 0 , followed by incubation with $10 \mu \mathrm{g} / \mathrm{ml}$ peptide-pulsed artificial antigen-presenting cell (aAPCs) (1x10 cells) on days 7 and 14 . Peptide specificity was assessed by interferon- $\gamma($ IFN- $\gamma)$ enzyme-linked immuno spot (ELISPOT) assay on day 21. (B) IFN- $\gamma$ ELISPOT assay was carried out (effector, $1 \times 10^{5}$ cells/well; target, $1 \times 10^{5}$ cells/well) in duplicate at least three times independently; representative data are shown. (C) T790M-A-specific CTLs of healthy donor 3 were incubated with $10 \mu \mathrm{g} / \mathrm{ml}$ peptide-pulsed T2 cells $(\mathrm{E}: \mathrm{T}=2: 1)$ for $3.5 \mathrm{~h}$ in the presence of an anti-human CD107a antibody. CD8 ${ }^{+} \mathrm{CD} 107 \mathrm{a}^{+}$cells were sorted using a FACSAria II cell sorter, which resulted in establishment of a T790M-A-specific CTL line.

CTLs specific for these peptides can be induced from human PBMCs. Given the effective induction of T790M-A (789-797) peptide-specific CTLs, we performed further analysis of the T790M-A peptide.

Generation of EGFR T790M-A-specific CTL line from human PBMCs. Next, we attempted to generate a purified T790M-A (789-797)-specific CTL line. Because the surface mobilization of CD107a is useful for identifying and isolating functional tumor-reactive $\mathrm{T}$ cells (25), we performed a CD107a assay to generate a purified T790M-A (789-797)-specific CTL line. Cultured cells stimulated by T790M-A peptide-pulsed DCs and aAPC-A2 in vitro were incubated with peptide-pulsed T2 cells at a ratio of $2: 1$ for $3.5 \mathrm{~h}$ at $37^{\circ} \mathrm{C}$ in the presence of an anti-CD107a antibody. More frequent $\mathrm{CD} 107 \mathrm{a}^{+}$cells were observed when CTLs were co-cultured with T790M-A peptide-pulsed T2 cells compared to HIV-peptide-pulsed $\mathrm{T} 2$ cells, and $\mathrm{CD} 8^{+} \mathrm{CD} 107 \mathrm{a}^{+}$ cells were sorted as a purified, peptide-specific CTL line using a FACSAria II cell sorter (Fig. 2C). A purified T790M-A-specific CTL line was established from healthy donor 3.
Cross-reactivity of the T790M-A-specific CTL line with other EGFR T790M-derived peptides. To assess its crossreactivity with other EGFR T790M-derived peptides, the T790M-A-specific CTL line was cultured with T2 cells pulsed with each peptide, and IFN- $\gamma$ production was measured by ELISPOT assay. The T790M-A-specific CTL line specifically recognized T2 cells pulsed with T790M-A (789-797) but not non-peptide-pulsed T2 cells. The T790M-A-specific CTL line did not recognize T2 cells pulsed with the T790M-A (789-797) wild-type (ITQLMPFGC) peptide. Also, T2 cells pulsed with T790M-B, -D, and -E were not recognized by the T790M-A-specific CTL line (Fig. 3A). However, the T790M-A-specific CTL line showed cross-reactivity with T2 cells pulsed with T790M-C.

Next, we evaluated the cytolytic activity of the T790M-Aspecific CTL line against cognate peptide-pulsed T2 cells. The T790M-A-specific CTL line specifically lysed T790M-A peptide-pulsed T2 cells but not HIV-peptide-pulsed T2 cells (Fig. 3B). These results suggest that the T790M-A-specific CTL line showed cross-reactivity against some EGFR 
A

Peptide(-)

T790M-A

T790M-B

T790M-C

T790M-D

T790M-E
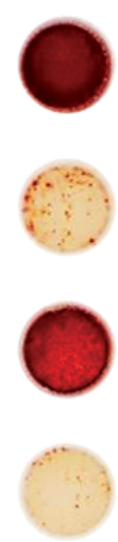

B

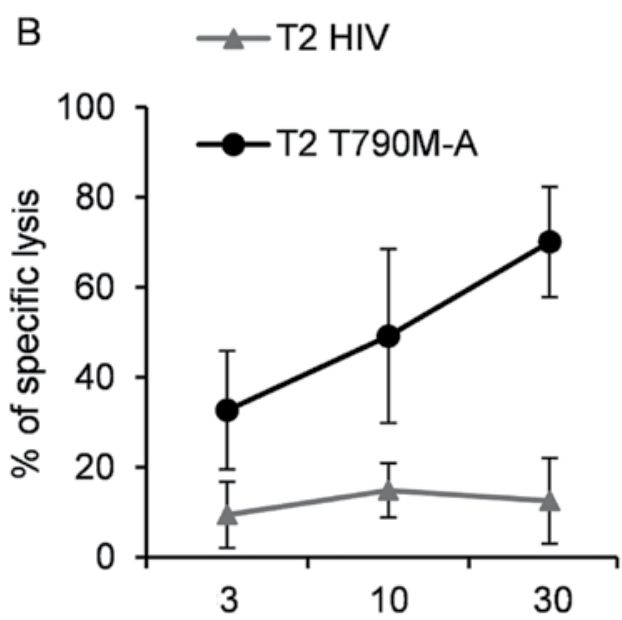

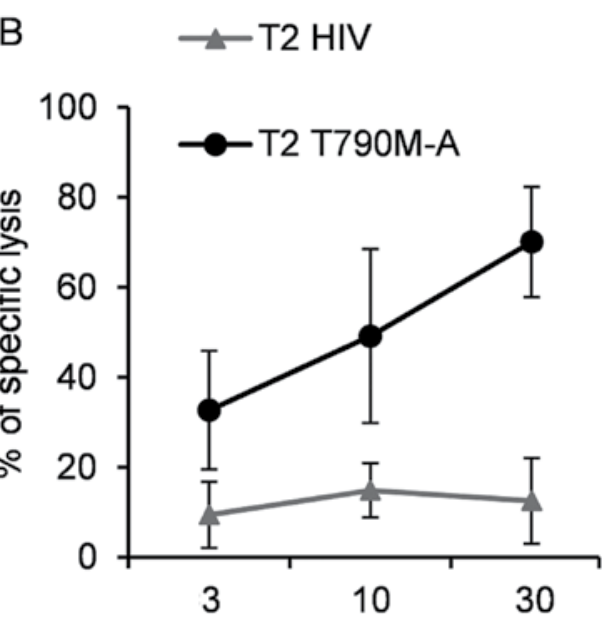

E:T ratio

T790M-Awt
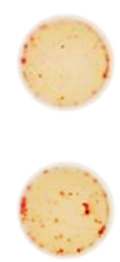

Figure 3. Cross-reactivity of the T790M-A-specific CTL line with threonine to methionine change at codon 790 of EGFR (EGFR T790M)-derived peptides. (A) Interferon- $\gamma($ IFN- $\gamma$ ) enzyme-linked immuno spot (ELISPOT) assay against T2 cells pulsed with each peptide. T2 cells pulsed with EGFR T790M-derived peptides (EGFR T790M-A, -B, -C, -D, -E, and Awt) were used as the target (effector $1 \times 10^{4}$ cells/well, target $1 \times 10^{4}$ cells/well). The assays were carried out in duplicate wells, and representative data are shown. (B) Cytotoxicity of the T790M-A-specific CTL line against T790M-A peptide-pulsed T2 cells. HIV-peptide-pulsed T2 cells were used as a negative control. Data are presented as means \pm SD of three independent batches.

T790M-derived peptides, but not the corresponding wild-type EGFR-derived peptide. This cross-reactivity seems to be favorable for efficacy against EGFR T790M+ cancer cells.

The T790M-A-specific CTL line recognizes and lyses $H L A-A 2^{+} T_{790 M^{+}}$NCSLC cells. Next, we assessed the ability of the T790M-A-specific CTL line to recognize the HLA-A2 ${ }^{+}$ $\mathrm{T} 790 \mathrm{M}^{+} \mathrm{NCSLC}$ cell line. This CTL line was incubated with 11-18 (T790M- ${ }^{-}$HLA-A2 ${ }^{+}$), T790M-A-pulsed 11-18, H-1975 $\left(\mathrm{T}_{790 M^{+}}\right.$HLA-A2 $^{-}$), or H-1975-A2 (T790M ${ }^{+}$HLA-A2 ${ }^{+}$), and IFN $-\gamma$ production was evaluated. We confirmed that the T790M-A-specific CTL line recognized peptide-pulsed 11-18 and H-1975-A2, but not 11-18 and H-1975, cells by IFN- $\gamma$ ELISPOT assay (Fig. 4A). Similar data were obtained using CTLs from healthy donor 1 stimulated with T790M-A peptide-pulsed DC and aAPC-A2 in vitro, which were not purified by the CD107a assay (data not shown).

To evaluate the function of the T790M-A-specific CTL line against H1975-A2, a CD107a assay was performed. CD107a ${ }^{+}$cells were detected more frequently in culture with H-1975-A2 than with H-1975 cells (Fig. 4B).

Finally, we investigated the cytotoxic activity of the T790M-A-specific CTL line against H-1975-A2. Target cells were labeled with calcein-AM and co-cultured with the effector cells for 4-6 h. The T790M-A-specific CTL line showed cytotoxic activity against H1975-A2 cells, but not H1975 cells (Fig. 4C). These results suggest that the T790M-A-specific CTL line can recognize NSCLC cells harboring the EGFR T790M mutation in an HLA-A2-restricted manner.

\section{Discussion}

Mutated antigens associated with tumor cell progression and survival or drug resistance represent novel targets for cancer vaccine therapy. Warren et al evaluated computationally the antigenic potential of somatic mutations that occur in human cancers (26). They showed that several gene mutation-derived epitopes have immunogenic potential, at least computationally. Moreover, point mutations within the ABL kinase domain of the $B C R-A B L$ gene are the most common causes of resistance to imatinib in chronic myeloid leukemia (CML) patients (27). Cai et al reported that the mutated $B C R-A B L$ gene was associated with a TKI-resistance-generated CTL epitope in CML patients (28). These results suggest new immunotherapeutic approaches based on a TKI-resistant mutation-derived neoantigen. That is, mutations associated with acquired resistance to TKI therapy can be targeted by immune-based treatment strategies. This strategy may be an option to treat the gene mutation-mediated drug-resistant cancer cells. In the present study, we demonstrated the immunogenicity of antigens from mutated EGFR that are involved in TKI resistance in NCSLC.

TAAs can be classified into several categories, such as cancer-testis (CT) antigens, overexpressed antigens, differentiation antigens, and mutated antigens. Of these, only mutated antigens are unique, because they are not expressed in normal tissues. Previous reports have shown that peptide vaccine therapy can occasionally induce ineffective CTL responses, contrary to expectations (29-31). One possibility is 

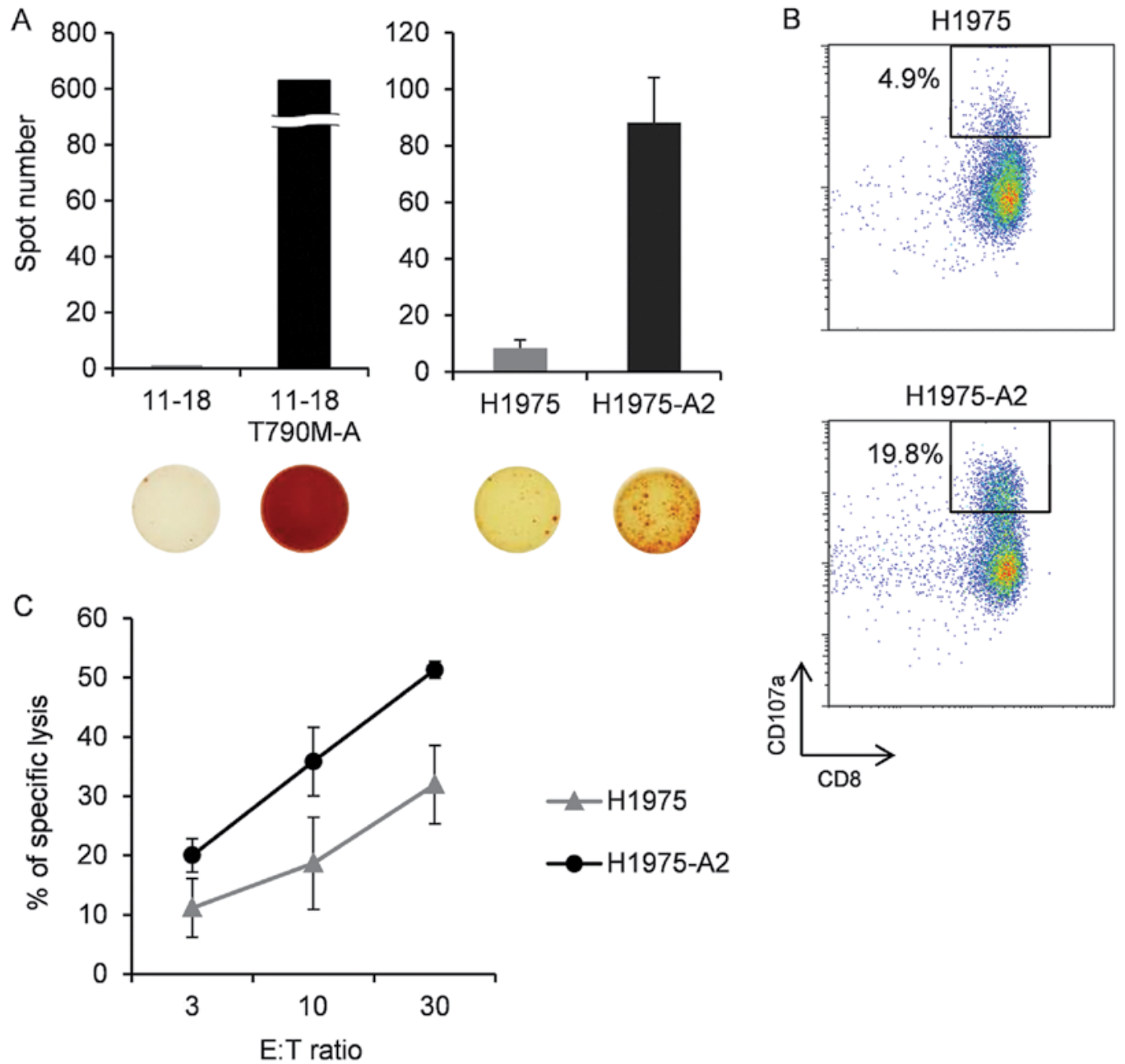

Figure 4. Reactivity of the T790M-A-specific CTL line against non-small-cell lung cancer (NSCLC) cells with or without the T790M mutation. (A) Interferon- $\gamma$ (IFN- $\gamma$ ) enzyme-linked immuno spot (ELISPOT) assay results for the T790M ${ }^{+}$and T790M- NSCLC lines. Left: 11-18 and T790M-A pep-

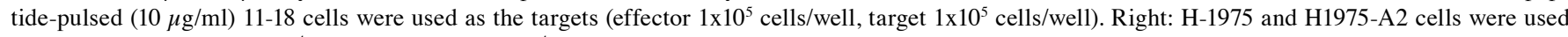
as the targets (effector, $5 \times 10^{4}$ cells/well; target, $5 \times 10^{4}$ cells/well). The bars indicate the IFN- $\gamma$ ELISPOT counts. (B) CD107a assay of the T790M ${ }^{+}$NSCLC $^{-1}$ line (E:T $=2: 1)$. CD8 ${ }^{+} \mathrm{CD} 107 \mathrm{a}^{+}$cells were gated. (C) Cytotoxicity against the $\mathrm{T} 700 \mathrm{M}^{+} \mathrm{NSCLC}$ cell line at the indicated effector/target ratios. Data are presented as means $\pm \mathrm{SD}$ of three independent batches.

A
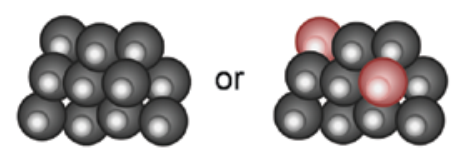

EGFR-TKIs

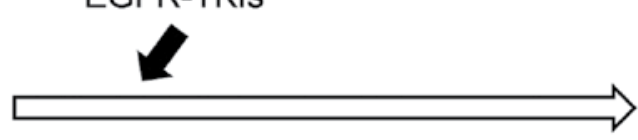

Peptide vaccine

$+\quad$ or

$+\quad$ or

Resistance

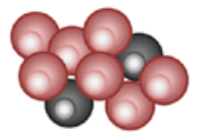

EGFR-TKls
B
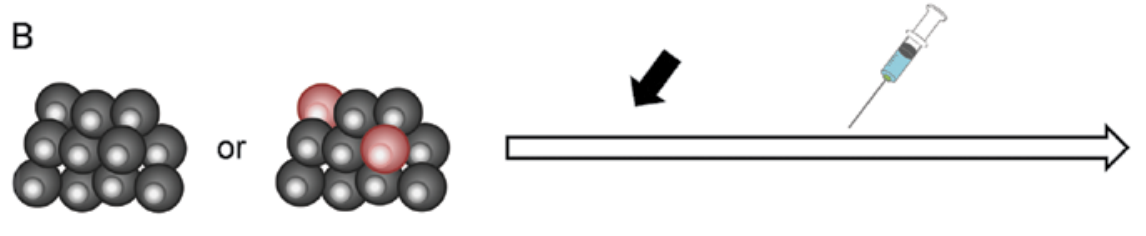

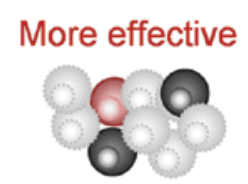

EGFR-TKI sensitivity cancer cells

EGFR-TKI resistant EGFR T790M ${ }^{+}$cancer cells

Figure 5. Combination therapy of epidermal growth factor receptor-tyrosine kinase inhibitor (EGFR-TKI) with T790M-targeted immunotherapy. (A) Generally, cancer cells develop acquired resistance to EGFR-TKI. (B) TKI-resistant cells harboring the T790M mutation were targeted by immunotherapy. This combination therapy may be effective against cancers with and without the threonine to methionine change at codon 790 of EGFR (EGFR T790M) mutation. 
that the induced antigen-specific CTLs have a low affinity, and thus recognize only target cells pulsed with high concentrations of the peptide and not naturally presented epitopes on tumor cells. Several EGFR-derived CTL epitopes have been identified $(32,33)$; however, the frequency of high-avidity EGFR-specific CTLs seems to be low in patients with EGFR-expressing cancers, because EGFR is a self-antigen that induces tolerance. The ability of low-avidity CTLs to recognize antigen-expressing tumor cells is considered to be weak. However, mutation-derived antigens are not self-antigens; thus, they would not be expected to induce immunotolerance, and so may have high immunogenicity. Indeed, in melanoma patients who experienced dramatic therapeutic effects after adoptive cell therapy with tumor-infiltrating lymphocytes (TILs), the mutated antigen-derived epitope was immunodominant and was recognized by tumor-reactive T cells $(34,35)$.

In the present study, BIMAS was used to select EGFR T790M-derived candidate peptides that bind to HLA-A*02:01 according to computer algorithms, and T790M-A-specific CTLs could be induced from PBMCs of all four healthy donors by stimulation with peptide-pulsed DCs and aAPCs. Amino acid substitution of anchor residues (at position 2 and the C-terminus for HLA-A2) can alter the binding affinity (36-38). Leucine and methionine are the preferred anchor residues at position 2 of HLA-A2 (36,37). T790M-A (IMQLMPFGC) harbors a substitution of threonine to methionine at the anchor site, which confers immunogenicity. Also, valine and leucine are the preferred anchor residues at the C-terminus (36,37).

Then, we designed the modified peptides, T790M-D (IMQLMPFGV, substitution of cysteine to valine at the C-terminus) and T790M-E (IMQLMPFGL, substitution of cysteine to leucine at the $\mathrm{C}$-terminus). These peptides bound to the HLA-A*02:01 molecule strongly (Fig. 1), but could not induce specific CTLs. T790M-D and -E are not self-antigens, being similar in this respect to T790M-A; this difference may be due in part to the difference in the frequency of peptide-specific CTL precursors. To confirm that the predicted candidate peptides are naturally presented peptides on tumor cells, peptide-specific CTL clones or lines induced by the peptides must recognize the tumor cells. A mass spectrometry (MS)-based method facilitates identification of peptide presentation by tumor cells (39). In this study, we confirmed the peptide-specific recognition of tumor cells by a peptide-specific CTL line, but not a CTL clone. However, CTL lines may contain distinct CTL clones that recognize irrelevant peptides, leading to apparent tumor reactivity (40). To avoid misleading tumor recognition and to evaluate the antigen-specific response of a CTL line, we used a peptide-specific CTL line established by CD107a sorting. An IFN- $\gamma$ ELISPOT assay suggested that the specific CTL line recognized NSCLC cells harboring the EGFR T790M mutation in an HLA-A*02:01-restricted manner.

The T790M-A-specific CTL line did not show activity against the corresponding wild-type peptide. This suggests that EGFR T790M-targeted immunotherapy has no effect on NSCLC prior to EGFR-TKI treatment, with the exception of any pre-existing population of T790M-harboring cells, at least theoretically. Thus, consideration of combination therapy, EGFR-TKI and EGFR T790M-targeted immunotherapy, seems reasonable. Several studies have suggested that combination therapy could improve the efficacy of cancer immunotherapy. For instance, some chemotherapeutic agents can lead to upregulation of TAA expression or improvement of tumor cell resistance to specific CTLs (41). Use of an EGFR-TKI or anti-EGFR antibody augments the IFN- $\gamma$-induced expression of MHC classes I and II by A431 malignant human keratinocytes (42). Moreover, gefitinib improved the cytotoxic activity of natural killer cells against H1975 by modulating the interaction between NK cells and cancer cells, and by inhibiting STAT3 expression (43). These results indicate that the combination of EGFR-TKI and immunotherapy may have synergistic activity against NSCLC cells. The concept of combination therapy is shown in Fig. 5. Adding EGFR T790M-targeted immunotherapy to EGFR-TKI treatment could control the progression of cancer cells harboring T790M.

Yamada et al reported two HLA-A2-restricted EGFR T790M-derived CTL epitopes (790-799 MQLMPFGCLL and 788-798 LIMQLMPFGCL) (44). In addition to these epitopes, we identified the HLA-A*02:01-restricted CTL epitope T790M-A (789-797 IMQLMPFGC). We found that a T790M-A-specific CTL line established from human PBMCs had the ability to recognize and lyse the HLA-A*02:01 ${ }^{+} \mathrm{T}^{*} 90 \mathrm{M}^{+}$NCSLC cell line, and importantly, did not show cross-reactivity with the corresponding wild-type EGFR peptide. These results suggest that the EGFR T790M-A-specific CTL line recognizes single amino acid substitutions, leading to a low level of auto-immune reaction. The combination of an EGFR-TKI and T790M-targeted immunotherapy may be useful for treatment of NSCLC with the T790M mutation.

\section{Acknowledgements}

We thank Professor Seiji Yano for providing the NSCLC cell line H1975, Dr Tetsuro Sasada for providing the NSCLC cell line H1975-A2, and Professor Naoto Hirano for providing aAPC-A2. This study was supported in part by the National Cancer Center Research and Development Fund (25-A-7), as well as Research for the Promotion of Cancer Control Programmes, Research on Applying Health Technology, and Third Term Comprehensive Control Research for Cancer from the Ministry of Health, Labour, and Welfare, Tokyo, Japan.

\section{References}

1. Jemal A, Siegel R, Ward E, Murray T, Xu J and Thun MJ: Cancer statistics, 2007. CA Cancer J Clin 57: 43-66, 2007.

2. Youlden DR, Cramb SM and Baade PD: The international epidemiology of lung cancer: geographical distribution and secular trends. J Thorac Oncol 3: 819-831, 2008.

3. Normanno N, Maiello MR and De Luca A: Epidermal growth factor receptor tyrosine kinase inhibitors (EGFR-TKIs): simple drugs with a complex mechanism of action? J Cell Physiol 194: 13-19, 2003.

4. Hynes NE and Lane HA: ERBB receptors and cancer: the complexity of targeted inhibitors. Nat Rev Cancer 5: 341-354, 2005.

5. Mok TS, Wu YL, Thongprasert S, et al: Gefitinib or carboplatinpaclitaxel in pulmonary adenocarcinoma. N Engl J Med 361: 947-957, 2009.

6. Mitsudomi T, Morita S, Yatabe Y, et al: Gefitinib versus cisplatin plus docetaxel in patients with non-small-cell lung cancer harbouring mutations of the epidermal growth factor receptor (WJTOG3405): an open label, randomised phase 3 trial. Lancet Oncol 11: 121-128, 2010. 
7. Maemondo M, Inoue A, Kobayashi K, et al: Gefitinib or chemotherapy for non-small-cell lung cancer with mutated EGFR. N Engl J Med 362: 2380-2388, 2010.

8. Zhou C, Wu YL, Chen G, et al: Erlotinib versus chemotherapy as first-line treatment for patients with advanced EGFR mutation-positive non-small-cell lung cancer (OPTIMAL, CTONG-0802): a multicentre, open-label, randomised, phase 3 study. Lancet Oncol 12: 735-742, 2011.

9. Rosell R, Carcereny E, Gervais R, et al: Erlotinib versus standard chemotherapy as first-line treatment for European patients with advanced EGFR mutation-positive non-small-cell lung cancer (EURTAC): a multicentre, open-label, randomised phase 3 trial. Lancet Oncol 13: 239-246, 2012.

10. Pao W, Miller VA, Politi KA, et al: Acquired resistance of lung adenocarcinomas to gefitinib or erlotinib is associated with a second mutation in the EGFR kinase domain. PLoS Med 2: e73, 2005.

11. Kobayashi S, Boggon TJ, Dayaram T, et al: EGFR mutation and resistance of non-small-cell lung cancer to gefitinib. N Engl J Med 352: 786-792, 2005

12. Fujita Y, Suda K, Kimura H, et al: Highly sensitive detection of EGFR T790M mutation using colony hybridization predicts favorable prognosis of patients with lung cancer harboring activating EGFR mutation. J Thorac Oncol 7: 1640-1644, 2012.

13. Su KY, Chen HY, Li KC, et al: Pretreatment epidermal growth factor receptor (EGFR) T790M mutation predicts shorter EGFR tyrosine kinase inhibitor response duration in patients with non-small-cell lung cancer. J Clin Oncol 30: 433-440, 2012.

14. Rosell R, Molina MA, Costa C, et al: Pretreatment EGFR T790M mutation and BRCA1 mRNA expression in erlotinib-treated advanced non-small-cell lung cancer patients with EGFR mutations. Clin Cancer Res 17: 1160-1168, 2011.

15. Shepherd FA, Douillard JY and Blumenschein GR Jr: Immunotherapy for non-small cell lung cancer: novel approaches to improve patient outcome. J Thorac Oncol 6: 1763-1773, 2011.

16. Rosenberg SA, Yang JC and Restifo NP: Cancer immunotherapy: moving beyond current vaccines. Nat Med 10: 909-915, 2004.

17. Kawakami Y, Wang X, Shofuda $\mathrm{T}$, et al: Isolation of a new melanoma antigen, MART-2, containing a mutated epitope recognized by autologous tumor-infiltrating $\mathrm{T}$ lymphocytes J Immunol 166: 2871-2877, 2001.

18. Lennerz V, Fatho M, Gentilini C, et al: The response of autologous $\mathrm{T}$ cells to a human melanoma is dominated by mutated neoantigens. Proc Natl Acad Sci USA 102: 16013-16018, 2005.

19. Yoshikawa T,Nakatsugawa M,Suzuki S, et al: HLA-A2-restricted glypican-3 peptide-specific CTL clones induced by peptide vaccine show high avidity and antigen-specific killing activity against tumor cells. Cancer Sci 102: 918-925, 2011.

20. Valmori D, Fonteneau JF, Lizana CM, et al: Enhanced generation of specific tumor-reactive CTL in vitro by selected Melan-A/MART-1 immunodominant peptide analogues. J Immunol 160: 1750-1758, 1998.

21. Parkhurst MR, Salgaller ML, Southwood S, et al: Improved induction of melanoma-reactive CTL with peptides from the melanoma antigen gp100 modified at HLA-A*0201-binding residues. J Immunol 157: 2539-2548, 1996.

22. Fong L, Hou Y, Rivas A, et al: Altered peptide ligand vaccination with Flt3 ligand expanded dendritic cells for tumor immunotherapy. Proc Natl Acad Sci USA 98: 8809-8814, 2001.

23. Hirano N, Butler MO, Xia Z, et al: Engagement of CD83 ligand induces prolonged expansion of $\mathrm{CD}^{+} \mathrm{T}$ cells and preferential enrichment for antigen specificity. Blood 107: 1528-1536, 2006.

24. Yoshimura M, Tada Y, Ofuzi K, Yamamoto $M$ and Nakatsura T: Identification of a novel HLA-A*02:01-restricted cytotoxic T lymphocyte epitope derived from the EML4-ALK fusion gene. Oncol Rep 32: 33-39, 2014.

25. Rubio V, Stuge TB, Singh N et al: Ex vivo identification, isolation and analysis of tumor-cytolytic T cells. Nat Med 9: 1377-1382, 2003.
26. Warren RL and Holt RA: A census of predicted mutational epitopes suitable for immunologic cancer control. Hum Immunol 71: 245-254, 2010.

27. Soverini S, Hochhaus A, Nicolini FE, et al: BCR-ABL kinase domain mutation analysis in chronic myeloid leukemia patients treated with tyrosine kinase inhibitors: recommendations from an expert panel on behalf of European LeukemiaNet. Blood 118: 1208-1215, 2011

28. Cai A, Keskin DB, DeLuca DS, et al: Mutated BCR-ABL generates immunogenic T-cell epitopes in CML patients. Clin Cancer Res 18: 5761-5772, 2012.

29. Yamshchikov GV, Barnd DL, Eastham S, et al: Evaluation of peptide vaccine immunogenicity in draining lymph nodes and peripheral blood of melanoma patients. Int J Cancer 92: 703-711, 2001.

30. Chen W, Yewdell JW, Levine RL and Bennink JR: Modification of cysteine residues in vitro and in vivo affects the immunogenicity and antigenicity of major histocompatibility complex class I-restricted viral determinants. J Exp Med 189: 1757-1764, 1999.

31. Meadows L, Wang W, den Haan JM, et al: The HLA-A*0201-restricted $\mathrm{H}-\mathrm{Y}$ antigen contains a posttranslationally modified cysteine that significantly affects T cell recognition. Immunity 6 : 273-281, 1997.

32. Shomura H, Shichijo S, Matsueda S, et al: Identification of epidermal growth factor receptor-derived peptides immunogenic for HLA-A2(+) cancer patients. Br J Cancer 90: 1563-1571, 2004.

33. Shomura H, Shichijo S, Komatsu N, et al: Identification of epidermal growth factor receptor-derived peptides recognised by both cellular and humoral immune responses in HLA-A24 non-small cell lung cancer patients. Eur J Cancer 40: 1776-1786, 2004.

34. Lu YC, Yao X, Li YF, et al: Mutated PPP1R3B is recognized by T cells used to treat a melanoma patient who experienced a durable complete tumor regression. J Immunol 190: 6034-6042, 2013.

35. Robbins PF,Lu YC,El-Gamil M, et al: Mining exomic sequencing data to identify mutated antigens recognized by adoptively transferred tumor-reactive T cells. Nat Med 19: 747-752, 2013.

36. Falk K, Rötzschke O, Stevanović S, Jung G and Rammensee HG: Allele-specific motifs revealed by sequencing of self-peptides eluted from MHC molecules. Nature 351: 290-296, 1991.

37. Sidney J, Southwood S, Mann DL, Fernandez-Vina MA, Newman MJ and Sette A: Majority of peptides binding HLA-A*0201 with high affinity crossreact with other A2-supertype molecules. Hum Immunol 62: 1200-1216, 2001.

38. Matsushita H, Vesely MD, Koboldt DC, et al: Cancer exome analysis reveals a T-cell-dependent mechanism of cancer immunoediting. Nature 482: 400-404, 2012.

39. Schirle M, Keilholz W, Weber B, et al: Identification of tumor-associated MHC class I ligands by a novel T cell-independent approach. Eur J Immunol 30: 2216-2225, 2000.

40. Parkhurst MR, Riley JP, Igarashi T, Li Y, Robbins PF and Rosenberg SA: Immunization of patients with the hTERT:540-548 peptide induces peptide-reactive $\mathrm{T}$ lymphocytes that do not recognize tumors endogenously expressing telomerase. Clin Cancer Res 10: 4688-4698, 2004.

41. Matar P, Alaniz L, Rozados V, et al: Immunotherapy for liver tumors: present status and future prospects. J Biomed Sci 16 : 30, 2009.

42. Pollack BP, Sapkota B and Cartee TV: Epidermal growth factor receptor inhibition augments the expression of MHC class I and II genes. Clin Cancer Res 17: 4400-4413, 2011.

43. He S, Yin T, Li D, et al: Enhanced interaction between natural killer cells and lung cancer cells: involvement in gefitinib-mediated immunoregulation. J Transl Med 11: 186, 2013.

44. Yamada T, Azuma K, Muta E, et al: EGFR T790M mutation as a possible target for immunotherapy; identification of HLA-A*0201-restricted T cell epitopes derived from the EGFR T790M mutation. PLoS One 8: e78389, 2013. 